Meta

Journal des traducteurs

Translators' Journal

\title{
Traduction automatique et classes d'objets : le problème de " porter un vêtement " en français et en coréen
}

\section{Man-Ghyu Pak}

Volume 42, numéro 1, mars 1997

Lexicologie et terminologie

URI : https://id.erudit.org/iderudit/003778ar

DOI : https://doi.org/10.7202/003778ar

Aller au sommaire du numéro

Éditeur(s)

Les Presses de l'Université de Montréal

ISSN

0026-0452 (imprimé)

1492-1421 (numérique)

Découvrir la revue

Citer cet article

Pak, M.-G. (1997). Traduction automatique et classes d'objets : le problème de " porter un vêtement » en français et en coréen. Meta, 42(1), 155-167.

https://doi.org/10.7202/003778ar
Résumé de l'article

Nous traitons, dans cet article, d'un des problèmes les plus difficiles dans le domaine de la traduction automatique, et qui n'est pas résolu à l'heure actuelle. C'est le cas où un verbe de la langue source correspond à plusieurs verbes de la langue cible. Nous présentons ici une solution qui s'appuie sur la classe d'objets, introduite par G. Gross pour décrire les traits sémantiques des noms et des verbes des langues naturelles : elle consiste à construire les classes d'objets tant dans la langue source que dans la langue cible et à les relier. 


\title{
TRADUCTION AUTOMATIQUE ET CLASSES D'OBJETS : LE PROBLĖME DE PORTER UN VETTEMENTEN FRANÇAIS ET EN CORÉEN'
}

MAN-GHYU PAK

Université de Kwandong, Kangnüng, Corée du Sud

\begin{abstract}
Résumé
Nous traitons, dans cet article, d'un des problèmes les plus difficiles dans le domaine de la traduction automatique, et qui n'est pas résolu à l'heure actuelle. C'est le cas où un verbe de la langue source correspond à plusieurs verbes de la langue cible. Nous présentons ici une solution qui s'appuie sur la classe d'objets, introduite par G. Gross pour décrire les traits sémantiques des noms et des verbes des langues naturelles: elle consiste à construire les classes d'objets tant dans la langue source que dans la langue cible et à les relier.
\end{abstract}

\begin{abstract}
This work deals with an unsolved problem that cannot be tackled in any systematic way: the case of tranislation where a lexical element (e.g. a verb) of the source language corresponds to several lexical elements of the target language. We offer a solution based on "classes d'objets", a system introduced by G. Gross to describe the semantic features of nouns and verbs in natural languages. Our solution consists in setting up the classes d'objets both in the source language and the target language and then linking them.
\end{abstract}

\section{한국어 요약}

본 논문은 지금까지 체계적인 방식으로 다룰 수 없는 것으로 알려져 온 기계번 역의 한 문제를 다룬다. 그것은 출발언어의 한 어휘요소(여기서는 동사)가 목표언어의 여 러 어휘요소에 대용되는 경우, 예킨대 불어의 porter (un vêtement)를 그 목적어 명사미 분포에 따라 '(바지를) 입다', '(신을) 신다', '(모자를) 쓰다' 따위로 기계로 하여금 정확 5) 번역시키려 할 때 제기되는 문제다. 여기서 우리는, 자연어의 명사와 동사의 의미자질올 기술하기 위해 가스통 그로스에 의해 도입된 체계인 classes d'objets를 이용한 해결책울 제시한다. 그것의 개요는, 먼저 두 언어의 classes d'objets를 작성한 후 이를 연결시키크 방법이다.

0. Aujourd'hui, la traduction automatique paraît stagner. L'une des raisons principales en est qu'il est très difficile de bien traiter les propriétés compliquées des langues naturelles, dont la plus difficile est la polysémie. De ce point de vue, il n'est pas abusif d'affirmer que le succès de la traduction automatique dépend de celui du traitement de la polysémie. La méthode dont on se sert actuellement pour lever les ambiguités des mots polysémiques est la notion de traits syntaxiques tels que humain, concret, abstrait, etc. Ceux-ci sont certes utiles: les traits concret et abstrait permettent de donner une bonne traduction à deux emplois différents du verbe français porter et de traduire porter par to bear en cas de complément abstrait et par to carry și l'objet est concret, comme on peut le voir : 
avec un objet abstrait dans :

Luc porte un nom français

Luc bears a french name

avec un nom concret dans :

Luc porte une valise

Luc carries a case

Cependant, ces traits sont insuffisants, n'apportant pas les informations indispensables à la reconnaissance automatique des différents emplois de porter, dans la mesure où ces traductions ne valent pas pour tous les abstraits et tous les concrets. Par exemple, un vêtement référant à un concret, le verbe porter qui lui est approprié ne se traduit pas par to carry mais par to wear:

Il porte des vêtements de tous les jours

He wears clothes for every day

Si le substantif vêtement est codé «concret» comme les termes valise ou sac, on comprend que cette seule spécification ne permette pas de sélectionner la bonne traduction en anglais. Pour prédire la traduction to wear, il est nécessaire d'ajouter le trait «vêtement». G. Gross appelle ces nouveaux traits sémantiques (établis sur la base des compatibilités entre opérateurs et arguments) des classes d'objets. Ce trait doit être noté pour chaque substantif désignant un vêtement. Le code, noté pour chaque élément de la classe, permet à un analyseur de prévoir la reconnaissance (ou la génération) de l'emploi et sa bonne traduction éventuelle)2 ${ }^{2}$.

Nous traitons, dans cet article, l'un des problèmes les plus difficiles dans le domaine de la traduction automatique et qui n'est pas résolu à l'heure actuelle. C'est le cas où un verbe de la langue source correspond à plusieurs verbes de la langue cible. Nous allons montrer que la notion de «classe d'objets» est toujours très utile dans la traduction automatique de ce genre de problèmes.

1. Chaque langue a sa propre manière de structurer le monde extérieur. Ainsi, une même action et un même état peuvent s'exprimer de façon différente selon les langues. La comparaison entre les langues permet de constater des divergences linguistiquement intéressantes.

Il arrive que deux sens reliés s'expriment au moyen de deux unités lexicales différentes dans une langue et par une même unité lexicale réalisée de deux façons dans l'autre. Par exemple, le français dispose du verbe mettre pour exprimer l'action de $<$ mettre un vêtement ${ }^{3}$ sur le corps $>$ et du verbe porter pour l'état résultant de l'accomplissement de l'action, tandis que le coréen utilise deux formes aspecto-temporelles d'un même verbe ip. Considérons les phrases françaises suivantes:

(1) a. Paul met un vêtement neuf sur lui

b. Paul porte un vêtement neuf sur lui

Les phrases (a) et (b) de (1) se traduisent respectivement en coréen par:

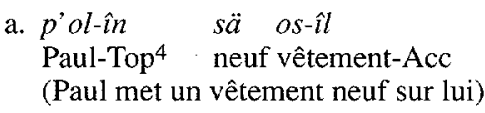

ip-nin-ta

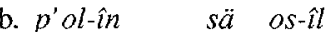
Paul-Top neuf vêtement-Acc
ip-ôss-ta
(Paul porte un vêtement neuf sur lui)


On peut voir dans ces exemples que mettre a pour équivalent coréen ip au présent, alors que porter a pour équivalent coréen ip au passé. La phrase coréenne ( 1 'b) donne lieu à une ambiguîté. Outre la première interprétation - celle de l'action passée: <Paul a mis un vêtement neuf sur lui> - elle a, selon les contextes, sa seconde interprétation, celle de l'état résultant de l'accomplissement de l'action: <Paul porte un vêtement neuf sur lui >5.

De plus, la forme mise au passé est paraphrasable par la forme complexe - ko iss (SVC suivi de l'auxiliaire), qui donne également lieu à l'interprétation statique. Ainsi la phrase coréenne $\left(1^{\prime} b\right)$ peut être réécrite ainsi :
(2) p'ol-în
$p^{\prime}$ ol-în
Paul-Top neuf vêtement-Acc
(Paul porte un vêtement neuf sur lui)
ip-ko iss-ta 6
mettre-SVC Aux-Déc

Le verbe français porter a donc un autre équivalent coréen, une autre forme aspectotemporelle du même verbe $i p^{7}$.

Un autre point intéressant est que le français utilise un même verbe (porter ou mettre) indépendamment du type de vêtements, tandis que le coréen dispose de différents verbes selon les cas. C'est-à-dire que le verbe français (porter ou mettre) correspond à plusieurs verbes en coréen. Considérons les phrases suivantes:

(3) Paul (met + porte) (une veste + un blouson)

Paul (met + porte) (un pantalon + un short)

Léa (met + porte) une jupe courte

Paul (met + porte) des caleçons plus chauds.

Paul (met + porte) (un gilet + un pardessus d'hiver)

(4) Paul (met + porte) un chapeau $(E+$ sur la tête)

Paul (met + porte) un masque

(5) Léa (met + porte) des bas très fins

Léa (met + porte) des chaussettes rouges

Il (met + porte) des souliers noirs

(6) Paul (met + porte) une montre $(\mathrm{E}+$ au poignet $)$

(7) Paul (met + porte) une cravate

(8) Paul (met + porte) une ceinture

(9) Léa (met + porte) une bague $(\mathrm{E}+$ au doigt $)$

Paul (met + porte) des gants ( $\mathrm{E}+$ aux deux mains)

Paul (met + porte) des lentilles de contact

(10) Paul (met + porte) des lunettes

(11) Léa (met + porte) des boucles d'oreilles

Léa (met + porte) un collier

(12) Léa (met + porte) un bracelet

(13) Paul (met + porte) une décoration ( $E+$ sur la poitrine)

Paul (met + porte) un badge $(E+$ sur la poitrine)

(14) Léa (met + porte) un foulard ( $E+$ au cou)

(15) Paul (met + porte) un brassard

Toutes les phrases (3) se traduisent par les phrases en ip, ce que nous avons déjà évoqué :
(3') p'ol-în
(côkoli + campa)-lîl
(ip-nîn-ta +ip-ôss-ta)
Paul-Top
(veste + blouson)-Acc
(mettre-Pré-Déc + mettre-Pas-Déc)
(Paul (met + porte) (une veste + un blouson))
p'ol-în
(paci + panpaci)-lîl
$(i p-n \hat{n}-t a+i p-\hat{s} s-t a)$
Paul-Top
(pantalon + short)-Acc
(Paul (met + porte) (un pantalon + un short))
(mettre-Pré-Déc + mettre-Pas-Déc)




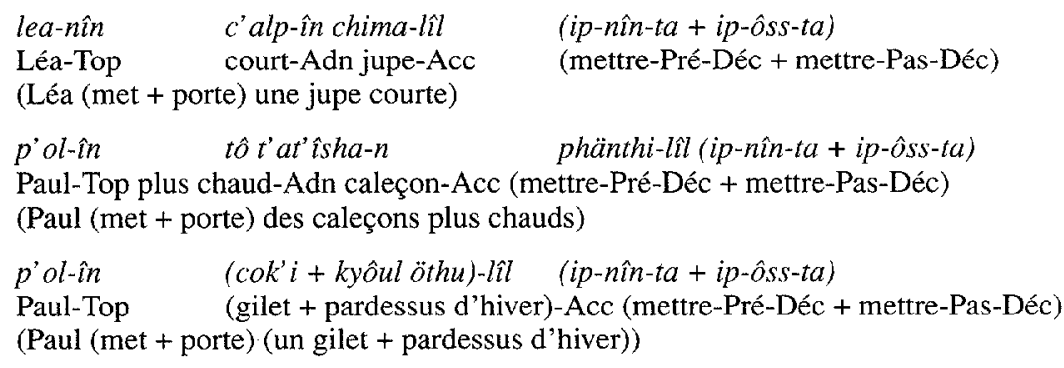

Mais les phrases en (4)-(15) ne se traduisent plus par des phrases en ip. En (4), le verbe porter (ou mettre, nous ne parlerons que de porter pour des raisons de commodité) se traduit par $s^{\prime} \hat{\imath}$ (se couvrir, se coiffer) :

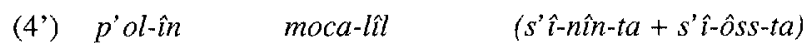
Paul-Top chapeau-Acc (se couvrir-Pré-Déc + se couvrir-Pas-Déc)
(Paul (met + porte) un chapeau sur la tête)
p'ol-în kamyôn-îl (s'înîn-ta $+s \hat{i}-\hat{o} s s-t a)$
Paul-Top masque-Acc (se couvrir-Pré-Déc + se couvrir-Pas-Déc)
(Paul (met + porte) un masque)

Dans (5), porter a comme équivalent coréen le verbe $\sin$ (chausser):

(5') lea-nîn yalp-în sîthakhing-îl (sin-nîn-ta $+\sin$-ôss-ta)

Léa-Top fin-Adn bas-Acc (chausser-Pré-Déc + chausser-Pas-Déc)

(Léa (met + porte) des bas très fins)

lea-nîn p'alkah-în yangmal-îl (sin-nîn-ta + sin-ôss-ta)

Léa-Top rouge-Adn chaussettes-Acc (mettre-Pré-Déc + mettre-Pas-Déc)

(Léa (met + porte) des chaussettes rouges)

$\begin{array}{lll}k \hat{i}-n i ̂ n & k \hat{m} m-\hat{i} n & k u t u-l \hat{l} l(\sin -n \hat{n} n-t a+\sin \text {-ôss-ta) } \\ \text { lui-Top } & \text { noir-Adn } & \text { chaussettes-Acc (mettre-Pré-Déc + mettre-Pas-Déc) } \\ \text { (Il (met + porte) des chaussettes noires) }\end{array}$

En (6) et (7), il se traduit respectivement par cha (mettre) et mä (nouer):
(6') p'ol-in (E+sonmok-e) sikye-lîl
Paul-Top poignet-à montre-Acc
(Paul (met + porte) une montre au poignet)
(cha-nîn-ta+cha-ôss-ta)
(mettre-Pré-Déc + mettre-Pas-Déc)

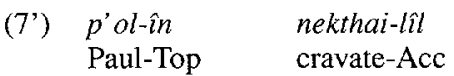
Paul-Top cravate-Acc.
(Paul (met + porte) une cravate)
$(m \ddot{a}-\hat{i} n-t a+m \ddot{a}-\hat{o} s s-t a)$
(nouer-Pré-Déc + nouer-Pas-Déc)

Pour (8), ces deux derniers verbes sont acceptés:
(8') p'ol-în
hôlit' i-lîl
Paul-Top ceinture-Acc
(Paul (met + porte) une ceinture)
$(c h a+m a ̈)-(n \hat{i n}-t a+\hat{o s s}-t a)$
(mettre + nouer)-(Pré-Déc + Pas-Déc)

Pour (9), on a $k^{\prime} i$ (insérer) :
(9) lea-nin
Léa-Top
$(E+$ sonkalak-e $)$
doigt-à
panci-îll ( $\left.k^{\prime} i-\hat{i n}-t a+k^{\prime} i-\hat{o} s s-t a\right)$ bague-Acc (insérer-Pré-Déc + insérer-Pas-Déc)
(Léa (met + porte) une bague au doigt) 


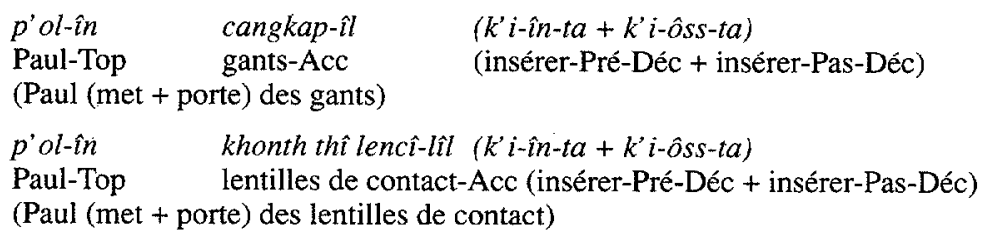
(10) p'ol-in ankyông-îl $\quad\left(k^{\prime} i+s^{\prime} \hat{\imath}\right)-(\hat{i} n-t a+\hat{o} s s-t a)$ Paul-Top lunettes-Acc (insérer + se couvrir)-(Pré-Déc + Pas-Déc) (Paul (met + porte) des lunettes)

Pour (11), on a ha (faire):
(11') lea-nîn
kwikôli-lîl
(ha-în-ta + ha-ôss-ta)
Léa-Top
boucles d'oreilles-Acc (faire-Pré-Déc + faire-Pas-Déc)
(Léa (met + porte) des boucles d'oreilles)

$\begin{array}{lll}\text { lea-nîn } & \text { mokkôli-lîl } & \text { (ha-în-ta }+ \text { ha-ôss-ta) } \\ \text { Léa-Top } & \text { collier-Acc } & \text { (faire-Pré-Déc + faire-Pas-Déc) } \\ \text { (Léa (met + porte) un collier) } & \end{array}$

Dans le cas de la phrase (12), les trois verbes cha, $k^{\prime} i$ et $h a$ peuvent être employés :
(12') lea-nîn
phalc' $i-l \hat{\imath} l$
$\left(c h a+k^{\prime} i+h a\right) \cdot(\hat{i n}-t a+\hat{o s s}-t a)$
Léa-Top
bracelet-Acc
(Léa (met + porte) un bracelet)
(mettre + insérer + faire)-(Pré-Déc + Pas-Déc)

Pour les phrases en (13), le verbe porter se traduit en tal (attacher):
(13') p'ol-in
kasîm-e huncang-îl
$(t a l-\hat{i n}-t a+t a l-o ̂ s s-t a)$
Paul-Top poitrine-à décoration-Acc
(attacher-Pré-Déc + attacher-Pas-Déc)
(Paul (met + porte) une décoration sur la poitrine)
p'ol-in kasimme ilimpho-lîl (tal-în-ta +tal-ôss-ta)
Paul-Top poitrine-à badge-Acc (attacher-Pré-Déc + attacher-Pas-Déc)
(Paul (met + porte) un badge sur la poitrine)

En (14), la traduction sera tuli (entourer) ${ }^{8}$ ou $h a$ (faire):
(14') lea-nin
Léa-Top
mok-e moktoli-lîl
cou-à foulard-Acc
(Léa (met + porte) un foulard au cou)
$(t u l \hat{l}+h a)-(\hat{i} n-t a+\hat{s} s s-t a)$
(entourer + faire)-(Pré-Déc + Pas-Déc)

Enfin, pour (15), on a cha, ha et $t u l \hat{\imath}^{9}$ :
(15') p'ol-în
wancang- $\hat{l}$
Paul-Top
brassard-Acc
(Paul (met + porte) un brassard)
$(c h a+h a+t u l \hat{l})-(\hat{\imath} n-t a+\hat{o s s}-t a)$ (mettre + faire + entourer)-(Pré-Déc + Pas-Déc)

On voit donc que le français a un verbe (porter ou mettre) dont le sens est très général, tandis que le coréen a plusieurs verbes qui dénotent une action très particulière, concrète et précise. Certes, en français aussi il y a des verbes dénotant une action plus concrète comme chausser, enfiler, nouer, épingler et boucler ${ }^{10}$ qui peuvent remplacer mettre (qu'il faut distinguer ici de porter ${ }^{11}$ ), mais ceux-ci ne constituent qu'un remplaçant partiel, à la différence du coréen. 
Par ailleurs, on pourrait envisager le verbe chakyongha comme un verbe globalisant. Mais nous ne croyons pas cela possible, car son niveau de langue est littéraire et il ne se combine pas avec tous les substantifs signifiant les vêtements. En effet, il n'est pas accepté par des noms tels que paci (pantalon), chima (jupe), sin (chaussures), yangmal (chaussettes), thal (masque), myônsapho (voile de mariée), etc. Il en ressort que le coréen n'a pas de verbe globalisant.

Il est évident que ces deux types de différences inter-langues posent beaucoup de difficultés lors de la traduction automatique, surtout lors de la description du dictionnaire de transfert bilingue (Bilingual Transfer Dictionary): on ne peut pas présenter, dans ce dictionnaire, une correspondance univoque entre deux termes simples. Dans le présent article, nous nous limiterons à l'étude des problèmes que pose le second type de divergence entre les deux langues.

Le verbe français porter a, comme équivalents coréens, neuf verbes différents au total, selon les cas.

Dans cette situation, il sera très intéressant de considérer les problèmes qui se posent lorsqu'on tente de traduire porter (un vêtement) en coréen et ses équivalents coréens en français.

La traduction du coréen en français peut être schématisée :

(16)

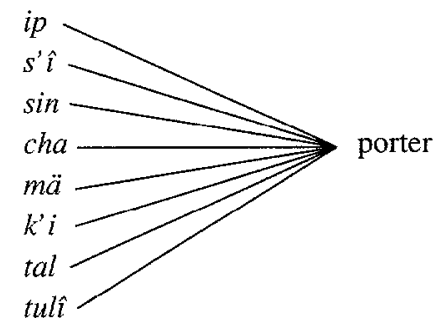

et la traduction du français en coréen peut s'écrire:

(17)

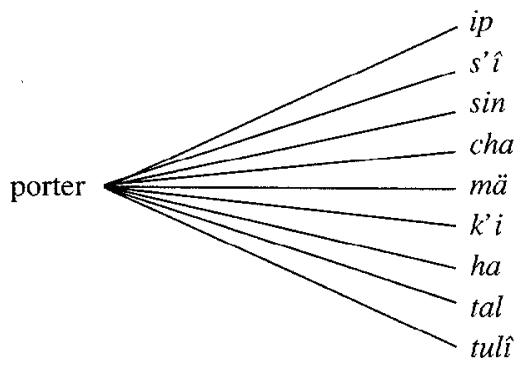

La traduction du type (16) ne pose pas de problèmes particuliers, à condition que la distinction entre les acceptions de chaque verbe coréen soit complètement établie ${ }^{12}$ : nous n'avons qu'à écrire dans le dictionnaire de transfert coréen-français :

(18) ip: porter

$s^{\prime} \hat{\imath}:$ porter

sin: porter

cha: porter

$m \ddot{a}:$ porter

$k^{\prime} i:$ porter

ha: porter

tal: porter

tulî : porter 
Le seul problème qui nous reste donc à résoudre est de savoir comment établir la distinction entre les différentes acceptions de chaque verbe polysémique du coréen. Nous croyons que, dans ce cas, la description au moyen des classes d'objets donne des résultats positifs dans ce type de traduction, car celles-ci contribueront à réduire les ambiguiités. La répartition des substantifs en classes d'objets est un système de description dans le cadre théorique du lexique-grammaire. Nous y reviendrons plus loin.

Considérons maintenant le cas de la traduction inverse, à savoir la traduction du français en coréen. Dans ce cas, des problèmes difficiles à traiter se posent. Si, dans le dictionnaire de transfert, on décrit tout simplement de la façon suivante:

(19) porter <vêtement > ip, s'î, sin, cha, mä, k'i, ha, tal, tulî

une seule des neuf traductions sera correcte dans la plupart des cas (on aura 3 formes correctes dans le meilleur des cas). Le problème est que les classes d'objets ne s'adaptent pas très bien dans ce type de traduction, parce qu'elles ne sont susceptibles de lever que les ambiguités de la langue source, non pas celles de la langue cible. Ce qui ne nous permet pas de choisir un équivalent adéquat parmi plusieurs candidats de la langue cible. Nous essaierons de faire une proposition pour résoudre ce problème.

2. Puisque la plupart des mots dans les langues naturelles sont polysémiques, on peut dire que la traduction a pour tâche de faire correspondre une des acceptions d'un verbe de la langue source (LS) à une des acceptions d'un verbe de la langue cible (LC). De ce point de vue, la description (18) n'est pas suffisante parce qu'elle ne lève pas l'ambiguïté éventuelle de chaque verbe coréen.

Pour G. Gross, une classe d'objets est définie par les relations syntaxiques qu'elle entretient avec une ou plusieurs classes de verbes appelés opérateurs appropriés. La classe d'objets <vêtement>, par exemple, est définie par le fait d'être sélectionnée en position $\mathbf{N}_{1}$ par les opérateurs appropriés porter $\mathbf{N}_{1}$, enlever $\mathbf{N}_{1}$, mettre $\mathbf{N}_{1}$ et changer de $\mathbf{N}_{1}$, dans l'état actuel de la description:

(20) Luc a (mis + enlevé) sa veste

Luc a changé de veste

Luc porte une veste ${ }^{13}$

D'après la recherche menée par l'équipe de G. Gross, environ 800 substantifs du français entrent dans la classe d'objets <vêtement> ainsi définie. Cette liste inclut non seulement le vêtement pris dans son sens général ou quotidien, mais également les accessoires, les parures et les objets tels que montre et lunettes, comme nous l'avons vu dans les exemples (1)-(15). Pour la langue française, ces derniers objets sont des vêtements.

La notion de classes d'objets se révèle d'une grande utilité dans le domaine de la traduction automatique, dans la mesure où elle permet de lever l'ambiguïté des mots polysémiques. Par exemple, on pourra présenter les traductions adéquates, en constituant l'article du verbe polysémique porter de la façon suivante:

(21) porter <vêtement $>$ : wear

porter $<$ blessure $>$ : bear

porter <partie du corps> : have

Ce qui amène les traductions suivantes:

(22) Luc porte un chapeau neuf $\rightarrow$ Luc is wearing a new hat

Luc porte des cicatrices $\rightarrow$ Luc bears scars

Le chameau porte deux bosses $\rightarrow$ The camel has two humps 
Considérons le problème de la constitution de la classe d'objets <vêtement> du coréen. Nous sommes tenté de la définir par la relation syntaxique suivante:

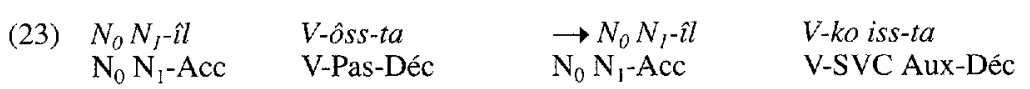

C'est-à-dire que sont retenus les substantifs qui occupent la position $\mathbf{N}_{1}{ }^{14}$. Comme nous l'avons dit plus haut, cette relation syntaxique caractérise les verbes dénotant le port du vêtement sur le corps.

La liste des vêtements du coréen établie au moyen de (23) recoupe quasiment celle du français établie par (20), comme on peut le voir dans les exemples (1)-(15). Seulement, les substantifs français tels que sac, malle, valise, serviette et cartable n'appartiennent pas à la classe du vêtement, tandis que leurs équivalents coréens y entrent sans aucun problème. Pour la langue coréenne, ces objets sont des vêtements.

Comme nous l'avons dit plus haut, chaque verbe coréen concerné est un mot polysémique. La classe d'objets <vêtement> permet de lever l'ambiguité. Par exemple, l'article du verbe $i p$, pourra se décrire de la façon suivante:

(24) ip <vêtement> : porter

ip $<$ faveur $>$ : recevoir

ip $<$ perte $>$ : subir

ip <blessure $>$ : recevoir, etc.

Mais la relation appropriée (23) ne suffit pas, telle quelle, car elle ne sépare pas les emplois des verbes coréens ip, sî, sin, cha, mä, $k^{\prime} i$, ha, tal et tulî. La classe établie par (23) doit donc être subdivisée. Pour la sous-classification, nous avons utilisé comme opérateurs appropriés, outre ces neuf verbes (correspondant à porter ou mettre), pôs, phul, p'ä (correspondant à enlever), kalaip, kalasin et kalak'i (correspondant à changer de). Nous présenterons ci-dessous un essai de description du dictionnaire de transfert coréen-français au moyen de sous-classes de <vêtement>. Ces sous-classes ne viennent pas d'études systématiques: elles ne sont qu'un exemple, constitué provisoirement pour des raisons de commodité 15 .

(25) ip <vêt.étr $>$, <tablier $>$ : porter

$s^{\prime} \hat{\imath}<$ chapeau $>$, <lunettes $>$, <masque $>$ : porter

$\sin <$ chaussures $>$, <chaussettes $>$ : porter

cha $<$ montre $>$, <ceinture $>$, <brassard $>$, <bracelet>, <banane>, <aff-per-mil >, <décoration $>$ : porter

$m \ddot{a}<$ cravate $>,<$ ceinture $>$ : porter

$k^{\prime} i<$ lunettes $>$, <lentilles de contact $>,<$ bague $>,<$ bracelet $>,<$ gants $>$ : porter

ha $<$ boucles d'oreilles $>$, <collier $>$, <bretelles $>$, <masque $>$, <brassard $>$, <bracelet $>$, $<$ banane $>,<$ foulard $>,<$ tablier $>$ : porter

tal $<$ décoration $>,<$ plaque d'identité $>$ : porter

tuli $<$ brassard $>$, <banane $>$, <foulard $>$, <tablier $>$ : porter ${ }^{16}$

où :
$<$ vêt.étr > =
vêtement au sens étroit, défini par les opérateurs appropriés: ip, pôs (enlever), kalaip (changer de) : la plupart des vêtements.
$<$ aff-per-mil $>=\begin{aligned} & \text { affaires personnelles militaires : chong (revolver), khal (canif), multhong } \\ & \text { (gourde), etc. }\end{aligned}$

3. Considérons maintenant le cas inverse, à savoir la traduction du français en coréen. Comme nous l'avons déjà remarqué, dans ce cas, il se pose des problèmes difficiles à traiter. $\mathrm{Si}$, dans le dictionnaire de transfert, on écrit tout simplement (19), une seule des 
neuf traductions sera correcte. Le problème est que les classes d'objets ne sont pas ici bien adaptées à la situation.

D'une part, c'est que les classes d'objets ne sont susceptibles de lever que les ambiguités de la LS, non pas celles de la LC. Ce qui ne nous permet pas de choisir un équivalent adéquat parmi plusieurs candidats de la LC.

D'autre part, dans l'état actuel de la description, le <vêtement> est la classe d'objets la plus petite, et même si celle-ci se subdivise plus en détail, il n'existe pas de garantie qu'elle recoupe exactement celle du coréen.

Cela est normal, dans la mesure où les classes d'objets sont définies de façon interne au système de la langue et à l'aide d'éléments lexicaux et non de traits abstraits ${ }^{17}$. En d'autres termes, les classes d'objets, se définissant par les comportements syntaxiques et reflétant donc la réalité d'une langue particulière, constituent une notion dépendante de cette langue. Il est bien entendu que pour faciliter la traduction, on ne peut modifier la description par les classes d'objets du français sur la base de celle du coréen.

Cette situation rend problématique la traduction du verbe porter: comment peut-on faire trouver par la machine les mots convenables dans chaque cas sans faire d'erreurs?

4. Dans cette situation, on peut envisager a priori trois solutions.

La première consisterait à renoncer au système des classes d'objets et à traduire <syntagme à syntagme>, comme on traite les expressions figées, à savoir traduire la séquence V N1 de la LS en V N1 de la LC. Voici quelques exemples :

(26) porter une veste : sangîi-lîl ip porter un chapeau : moca-lîl s' $\hat{\imath}$ porter des bas : sithakhing-îl sin porter une montre : sikye-lill cha

Cette méthode pose des problèmes non seulement du point de vue linguistique mais aussi informatique. Les séquences en (26) ne sont pas des expressions figées mais des expressions libres. De plus, cette méthode pose le problème de la multiplication des entrées, et il n'est pas économique de faire contrôler 800 séquences (substantifs) à la machine, surtout si l'on pense à l'ensemble des traductions de ce type.

La deuxième solution est d'établir un stade où la machine corrige les fautes commises lors de la traduction à l'appui du dictionnaire de transfert LS-LC. Cette méthode exige qu'on construise également un dictionnaire de la LC à côté du dictionnaire LS-LC. Par exemple, tout d'abord, on fait traduire par le système tous les porter (un vêtement) en ip parce que celui-ci convient pour le plus grand nombre de substantifs <vêtements>. Et ensuite, on fait corriger en se référant au dictionnaire LC, i.e. le dictionnaire coréen. Mais cette méthode présente toujours un problème difficile : comment fait-on corriger les séquences mal formées?

La troisième et dernière solution est de changer la méthode de description dans le dictionnaire de transfert en nous appuyant toujours sur le système des classes d'objets. L'essentiel est de construire les classes d'objets non seulement dans la LS mais encore dans la $\mathrm{LC}$ et de les relier. D'après cette méthode, la traduction se fera par les étapes suivantes:

1) faire passer le texte dans le dictionnaire de la LS où tous les substantifs sont codés par les classes d'objets et où tous les verbes sont codés par la liste des substantifs avec lesquels ils sont susceptibles de se combiner;

2) faire étiqueter le substantif par sa (ses) classe(s) d'objets et étiqueter le verbe par la liste des classes d'objets combinables avec lui, en se référant au dictionnaire de la LS;

3) faire lever l'ambiguité du verbe et reconnaître l'identité de l'acception en combinant le substantif et le verbe qui ont en commun la même classe d'objets; 
4) faire transférer le substantif étiqueté par sa classe d'objets dans l'équivalent de la LC étiqueté lui aussi par sa classe d'objets, en se référant au dictionnaire de transfert LS-LC, où tous les substantifs et tous les verbes sont codés par les classes d'objets; puis faire transférer le verbe codé par sa classe d'objets dans les verbes-candidats de la LC, chacun codé par sa classe d'objets en se référant au dictionnaire de transfert LS-LC;

5) faire traduire correctement en combinant le substantif et le verbe qui ont en commun la même classe d'objets.

Considérons, par exemple, le cas de la traduction de porter des chaussures de sport en coréen. Si l'on fait passer cette séquence dans le dictionnaire du français (LS) constitué sur la base de codes de classes d'objets, le substantif chaussures de sport recevra la marque correspondant à sa classe d'objets de la façon suivante :

(27) chaussures de sport: < vêtement>

et le verbe porter recevra la marque suivante:

(28) porterl : N1 =<vêtement> porter $2: \mathrm{Nl}=<$ blessure $>$ porter $3: \mathrm{N} 1=<$ partie du corps $>$

...

La machine lèvera l'ambiguïté du verbe et reconnaîtra l'identité de l'acception en combinant le substantif et le verbe porterl qui ont en commun la même classe d'objets <vêtement>.

D'autre part, on constitue l'article du substantif chaussures de sport du dictionnaire de transfert LS-LC de la façon suivante:

(29) chaussures de sport <vêtement> : untonghwa <chaussures>

La référence à (29) permet de transférer chaussures de sport en untonghwa, codé lui aussi par sa classe d'objets <chaussures $>$.

On constitue également l'article du verbe porter du dictionnaire de transfert LS-LC de la façon suivante:

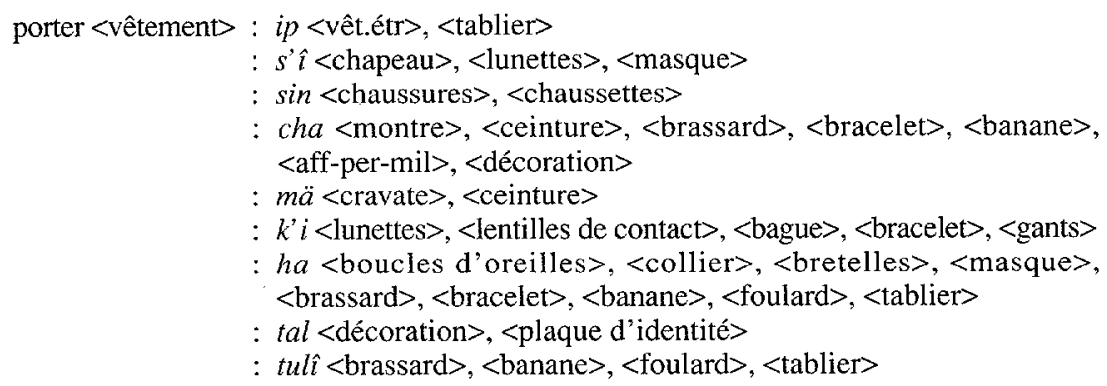

La référence à (30) fait transférer le verbe porter dans les verbes-candidats du coréen, chacun codé par sa classe d'objets.

Enfin, la machine arrive à traduire correctement en combinant le substantif untonghwa et le verbe sin, car ils ont en commun la même classe d'objets <chaussures>, à la suite de la programmation, comme on le voit dans ce qui suit:

(31) porter des chaussures de sport $\rightarrow$ untonghwa-lîl sin 
Parmi les trois solutions, nous croyons que la troisième est la meilleure puisqu'elle permet d'aboutir à la traduction exacte sans aucun problème. Du point de vue informatique, on peut facilement contrôler 800 substantifs par une trentaine de classes d'objets, et qui mieux est, cette méthode sera utile à toutes les traductions de ce type, i.e. dans le cas où un verbe de la LS correspond à plusieurs verbes de la LC. Son autre mérite est sa justesse du point de vue linguistique en ce qu'on peut garder le système de classes d'objets. Ajoutons, pour finir, que, par notre travail, nous avons pu vérifier une fois de plus l'utilité des classes d'objets dans la traduction automatique.

\section{Notes}

1. Je tiens à remercier Gaston Gross, Christian Leclère et Denis Le Pesant d'avoir lu le manuscrit et de m'avoir donné de précieux conseils.

2. G. Gross (1995: 83-84; et à paraitre : 1-4).

3. Nous n'entendons pas par <vêtement> son sens général ou quotidien, mais une classe d'objets définie de façon linguistique dont nous parlerons plus loin. Pour le moment, nous nous contenterons de dire que cette classe dépasse celle de la vie quotidienne, en ce qu'elle comprend des objets tels que parures et lunettes.

4. Nous nous référons au système de notation du L.A.D.L. Nous y ajoutons un certain nombre d'abréviations nécessaires à la description du coréen, ainsi qu'à la traduction mot à mot des exemples.

Les symboles et les abréviations utilisés dans cet article sont les suivants: Acc: Accusatif, Adn : Adnominatif, Aux : Auxiliaire, Top: Topique, Déc : Déclaratif, Pas : Passé, Pré : Présent, SVC : Suffixe Verbal Conjonctif.

5. Ce phénomène rappelle le cas des verbes tels que nîlk (vieillir) et cuk (mourir). Considérons les phrases:

(i) a. p'ol-în nîlk-ôss-ta

Paul-Top vieillir-Pas-Déc

(Paul est vieux)

b. p'ol-în cuk-ôss-ta

Paul-Top mourir-Pas-Déc

(Paul est mort)

Dans ces exemples, les formes verbales mises au passé dénotent un état qui résulte d'une action qu'il présuppose. On pourra se reporter à Pak (1993: 201-202).

6. L'interprétation de la forme -ko iss est a priori ambiguë. La première interprétation est stative, comme c'est le cas de (2). Nous donnons un autre exemple:

$$
\begin{array}{lll}
k \hat{\imath}-n \hat{n} & k \hat{\imath} i l-\hat{\imath} l & \text { al-ko iss-ta. } \\
\text { lui-Top ce affaire-Acc } & \text { savoir-SVC Aux-Déc } \\
\text { (ll est au courant de cette affaire) }
\end{array}
$$
Dans la seconde, l'action est en cours, comme dans :

$$
\begin{aligned}
& \text { lui-Top travail-Acc faire-SVC Aux-Déc } \\
& \text { (Il est en train de faire le travail) }
\end{aligned}
$$$$
\text { (iii) kînin il-ill ha-ko iss-ta. }
$$

L'interprétation dépend du sens de chaque verbe: en général, les verbes dénotant un état psychologique donnent lieu à la première interprétation, et les verbes dénotant une action concrète à la seconde. Or, pour ce qui est des verbes de port du vêtement, les deux interprétations sont possibles, selon les contextes.

7. Ce type de relation syntactico-sémantique n'est observable qu'avec les verbes dénotant le port du vêtement. Avec les autres verbes, les deux phrases ont des interprétations différentes, comme on peut le voir dans les exemples suivants:
(iv) $\quad p^{\prime}$ ol-în hösa-e ka-ass-ta aller-Pas-Déc
(v) p'ol-in p'ali-e sal-ass-to habiter-Pas-Déc
(vi) p'ol-in kî il-illal-ass-ta $\quad \neq$ savoir-Pas-Déc
$\neq \quad$ p'ol-in hösa-e ka-ko iss-ta aller-SVC Aux-Déc

\begin{tabular}{|c|c|c|c|}
\hline (vii) & $\begin{array}{l}p^{\prime} \text { ol-în nîlk-ôss-ta } \\
\text { vieillir-Pas-Déc }\end{array}$ & $\neq$ & $\begin{array}{l}p^{\prime} o l-\hat{n} n \text { nîlk-ko iss-ta } \\
\text { yieillir-SVC Aux-Déc }\end{array}$ \\
\hline (vi & $\begin{array}{l}p^{\prime} o l-\hat{n} n c u k-\hat{o} s s-t a \\
\text { mourir-Pas-Déc }\end{array}$ & $\neq$ & $\begin{array}{l}p^{\prime} o l-i n c u k-k o \text { iss-ta } \\
\text { mourir-SVC Aux-Déc }\end{array}$ \\
\hline
\end{tabular} p'ol-in pali-e sal-ko iss-ta habiter-SVC Aux-Déc p'olin ki il-il al-ko iss-ta

Les verbes tels que nîlk et $c u k$ ne présentent pas non plus cette propriété : 
8. Dans ce cas, on peut aussi employer le verbe kam (enrouler) au sens figuré, mais nous ne le classifions pas parmi les équivalents corćens de mettre ou porter, car son niveau de langue n'est pas standard et il a une connotation un peu péjorative, nous semble-t-il.

9. Parmi les substantifs qui prennent pour le verbe tuli, le substantif apchima (tablier) accepte également $i p$ :
(ix) lea-nin
apchima-tal
$(t u l \hat{t}+i p)-(\hat{m}-t a+\hat{o s s}-t a)$
Léa-Top tablier-Acc
(entourer + mettre)-(Pré-Déc + Pas-Déc)

(Léa (met + porte) un tablier)

Remarquons en passant que, dans ce cas, le verbe ha est aussi accepté, mais seulement comme traduction de porter (et pas de mettre) :
(x) lea-nin
apchima-lill
$h a-(* i n-t a+o ̂ s s-t a)$
Léa-Top tablier-Acc
faire-(Pré-Déc + Pas-Déc)

Si la phrase mise au présent est acceptée, on aura une interprétation atemporelle, et non celle du présent. Remarquons que la situation est la même pour le substantif sangpok (vêtement de deuil), à côté de ip, ha est accepté, mais pas comme traduction de mettre:
(xi) lea-nin sangpok-il
Léa-Top vêtement de deuil-Acc
$h a-(* i n-t a+\hat{o s s}-t a)$
(Léa (met + porte) le deuil)

Comme dans le cas de apchima, si la phrase mise au présent est acceptée, ce sera une interprétation atemporelle, et non pas celle du présent.

10. enfiler (une veste + un pantalon + des bas + des bottes)

nouer (une cravate + un foulard + un tablier)

épingler (des décorations + des badges) sur sa poitrine

boucler une ceinture autour de sa taille

11. Sauf que si l'on met au passé, on a, comme en coréen, une équivalence:
Paul a noué une cravate
$\simeq$
Paul porte une cravate
Paul a enfilé des bottes
$\simeq$
Paul porte des bottes

12. Chacun des verbes coréens concernés, lui aussi un mot polysémique, ne se traduit pas toujours par porter, plus précisément, il ne se traduit par porter que lorsque l'objet direct est un <vêtement>. Nous y reviendrons plus loin.

13. G. Gross (1992:8)

14. Dans ce cas, l'opérateur approprié est une relation transformationnelle.

15. Nous n'avons pas fait la subdivision de façon systématique, ce qui exige une étude à part. En effet, il convient, pour chaque sous-classe, d'examiner méthodiquement tout le lexique des noms.

16. Voici la liste des sous-classes de vêtement:

$<$ bague> : panci (bague), kyôlhon panci (bague de fiançaille), etc.

$<$ banane> : côntä (banane)

$<$ boucles d'oreilles>: kwikôli (boucles d'oreilles), etc.

$<$ bracelet $>$ : phalc'i (bracelet)

$<$ brassard > : wancang (brassard)

$<$ bretelles> : melp'ang (bretelles)

$<$ ceinture> : hôlit' $i$ (ceinture), hyôktä (ceinture), pelthî (ceinture), etc.

$<$ chapeau>: moca (chapeau), kas (chapeau traditionnel coréen), myônsapho (voile de mariée),

<chaussettes> : yangmal (chaussettes), pôsôn (chaussettes traditionnelles coréennes), sîthaking (bas), myônyangmal (chaussettes en coton), etc.

$<$ chaussures>: $\sin$ (chaussures), kutu (escarpins), untonghwa (chaussures de sport), canghwa (bottes),

komusin (chaussures en caoutchouc), cipsin (chaussures en paille), etc.

$<$ cravate> : nekthai (cravate), napi nekthai (nœud papillon)

$<$ collier $>$ : mokkôli (collier)

$<$ décoration> : huncang (décoration), etc.

$<$ foulards: moktoli (foulard), syol (châle), sîkaphî (écharpe), etc.

$<$ gants> : cangkap (gants), kacuk cangkap (gants en cuir), sukap (gants), etc.

<lentilles de contact>: khonth kthî lencî (lentilles de contact), hatî lencî (lentilles dures), sophithî lencî (lentilles souples), etc.

$<$ lunettes>: ankyông (lunettes), totpoki (loupe), etc.

$<$ masque> : thal (déguisement), masîkî (masque), pangtokmyôn (masque à gaz), etc.

$<$ montre> : sikye (montre), sonmok sikye (montre), cônca sikye (montre à quartz), kîmsikye (montre en or), etc. <plaque d'identités: ilîmphyo (badge portant le nom), myôngchal (badge portant le nom), kyekipcang (badge portant le grade militaire), etc.

$<$ tablier> : apchima (tablier)

17. G. Gross (1992: 16) 


\section{RÉFÉRENCES}

GROSS, Gaston (1992a) : «Forme d'un dictionnaire électronique», L'environnement traductionnel. La station de travail du traducteur de l'an 2001, Sillery, Presses de l'Université du Québec et AUPELF-UREF, pp. 255-271.

GROSS, Gaston (1992b) : «Classes d'objets et enseignement», Actes du colloque sur le traitement automatique, Séoul.

GROSS, Gaston (1994a): «Classes d'objets et synonymie», Annales littéraires de l'Université de Besançon, Série linguistique et sémiotique, vol. 23 , Besançon.

GROSS, Gaston (1994b): «Un outil pour le FLE: les classes d'objets», Actes du colloque du FLE, Lille, Presses Universitaires de Lille.

GROSS, Gaston (1994c) : «Classes d'objets et description des verbes», Langages, n, 115, Paris, Larousse.

GROSS, Gaston (1995): «Les classes d'objets», Les concepts opératoires de l'INaLF, rapport de I'INaLF, pp. 83-88.

GROSS, Gaston (à parâtre) : «Une sémantique nouvelle pour la traduction automatique : les classes d'objets».

LE PESANT, Denis (1994): «Les compléments nominaux du verbe <lire>, une illustration de la notion de <classe d'objets $>$, Langages, n 115 , Paris, Larousse.

LEHRBERGER, and BOURBEAU (1988): Machine Translation: Linguistic Characteristics of MT Systems and General Morphology of Education, Amsterdam, John Benjamins Publishing Co.

PAK, Manghyu (1993): Restructuration de la complétive des verbes de cognition et des verbes de communication en français et en coréen : lexique-grammaire comparé. Thèse de doctorat, Université Nationale de Séoul. 